\title{
The Effects of Listing Authors in Alphabetical Order: A Survey of the Empirical Evidence*
}

\author{
Matthias Weber \\ 10 November 2017 \\ CEFER, Bank of Lithuania \& Faculty of Economics, Vilnius University
}

\begin{abstract}
Each time researchers jointly write an article, a decision must be made about the order in which the authors are listed. There are two main norms for doing so. The vast majority of scientific disciplines use a contribution-based norm according to which authors who contributed the most are listed first. Very few disciplines, most notably economics, instead resort primarily to the norm of listing authors in alphabetical order. It has been argued that (i) this alphabetical norm gives an unfair advantage to researchers with last names starting with a letter early in the alphabet and that (ii) researchers are aware of this "alphabetical discrimination" and react strategically to it, for example by avoiding collaborations with multiple others. This article surveys the empirical literature and finds convincing evidence that alphabetical discrimination exists and that researchers react to it.
\end{abstract}

Keywords: Alphabetical discrimination; lexicographic order; contribution-based order; scientific publishing

\footnotetext{
* I would like to thank Rei Sayag, Michał Krawczyk, Patrick Grüning, and two anonymous reviewers for comments and suggestions. The views expressed herein are those of the author and do not necessarily reflect the views of the Bank of Lithuania. Email: mweber@lb.lt.
} 


\section{Introduction}

In most scientific disciplines, the order of authors on published articles depends on the relative contributions of these authors. The first author is the one who has contributed the most and other authors follow in descending order. An exception is the last position, which is often taken by the most senior author or the author with the overall coordination (for example the advisor in papers written jointly with graduate students or the head of the laboratory). However, partly authors are listed intentionally in alphabetical order (i.e., lexicographically). ${ }^{1}$ There is some alphabetical ordering in most disciplines (Levitt and Thelwall, 2013). However, across all disciplines, it applies to less than $4 \%$ of publications (Waltman, 2012). These publications are not randomly distributed across fields: There are very few fields in which alphabetical name ordering is the norm rather than the exception. The fields in which more than $50 \%$ of publications intentionally choose alphabetical name ordering are "Business and Finance", "Economics", "Mathematics", and "Physics, Particles and Fields" (Waltman, 2012; Frandsen and Nicolaisen, 2010; the latter uses the specification of "High Energy Physics" instead of "Physics, Particles and Fields").

Ordering authors on an article is necessary for the vast majority of scientific papers as the last decades have seen a sharp rise in the number of multi-authored publications. By now around $90 \%$ of all scientific publications have more than one author (the number of multi-authored publications in the few fields often using alphabetical name ordering is a bit lower, possibly due to the alphabetical name ordering norm; Abt, 1992; Frandsen and Nicolaisen, 2010; Hudson, 1996; Waltman, 2012). Note that it has been shown that multi-authored articles are more influential than single-authored articles (Wuchty et al., 2007; Gazni and Didegah, 2011).

\footnotetext{
1 "Intentional" alphabetical ordering refers to the number of publications with alphabetical ordering while correcting for the fact that, when adhering to the contribution-based rule, the order is sometimes also alphabetical (for more details, see Waltman, 2012). The number of actual alphabetical orders is naturally higher than this number, but it can be at most twice as high (the number of intentionally alphabetically ordered articles can be interpreted as the excess of alphabetically ordered articles over the number arising from all authors choosing the contribution-based approach).
} 
The question of whether the particular name ordering norm matters to scientists and that of what the most appropriate norm is has received considerable attention in the last decades. Most of the discussions have probably taken place outside of scientific journals, but also there the topic has been treated repeatedly (e.g. Zuckerman, 1968; Over and Smallman, 1973; Rudd, 1977; Garfield, 1997; Shevlin and Davies, 1997; Stubbs, 1997; Tregenza, 1997; many contributions are more recent and will be mentioned in the remainder). In such treatments, alphabetical name ordering has received a lot of criticism as many scientists consider it to give an unfair advantage to researchers with names early in the alphabet. The term "alphabetical discrimination" was coined for this.

There are multiple reasons why alphabetical name ordering can be considered unfair. Authors of articles with more than two authors disappear for example often in the term "et al." when references are abbreviated as "first author et al." (in particular in the social sciences). There is ample research showing that visibility and recognition are important (e.g., Goldstein and Gigerenzer, 2002; Serwe and Frings, 2006). Thus, alphabetical name ordering may lead to an increased visibility of researchers with last names early in the alphabet. Ray and Robson (2016) illustrate this nicely: "To add insult to injury, one of us (Ray) had just been enthusiastically recommended a 'wonderful paper' by Banerjee et al (2001), on which he was a co-author". A similar argument can be made for reference lists. When multiple references in a row start with the same name, it is likely that this author will be remembered as an expert in the field. If authors are listed in alphabetical order, this means that mainly authors with a name early in the alphabet will enjoy such recognition. Furthermore, if researchers in fields that mainly use alphabetical name ordering are not only evaluated by researchers in their own field, authors who are often listed as first authors will be evaluated better than authors who are not often first authors. ${ }^{2}$

\footnotetext{
${ }^{2}$ There are also reasons to believe that alphabetical name ordering contributes to discrimination against women and minorities (Sarsons, 2015) and that it advantages more senior authors (Lake, 2010). These arguments will not be discussed further in this survey.
} 
These arguments seem convincing. However, one could also argue that they do not matter in practice. One could even claim that an alphabetical ordering norm gives an advantage to people with last names late in the alphabet. Only they have the opportunity to signal particularly large contributions through deviating from the alphabetical norm, so the argument goes (see Engers et al., 1999).

Theoretical results are necessarily driven by the assumptions of the model. ${ }^{3}$ While one may find some arguments more and some less convincing, in the end the problem boils down to an empirical question. Are the disadvantages that authors toward the end of the alphabet have stronger or weaker than their advantages?

If alphabetical discrimination plays a role, or is presumed to do so, it is interesting to know whether researchers react to it strategically. An alphabetical norm could for example lead researchers with names late in the alphabet to cooperate less with other researchers. Some people may even manipulate their last names if they have the opportunity, for instance by using only one out of two last names.

In this survey, I review the empirical literature investigating these two closely related questions. The first is the question of whether and to which extent authors with a last name early in the alphabet have an advantage over authors with a name late in the alphabet under an alphabetical norm. The second is the question of whether and how researchers react strategically to an alphabetical norm. The relevant studies focus mainly on economics as the primary field of alphabetical name ordering (although even in this field, a considerable share of articles is not ordered alphabetically). This

\footnotetext{
${ }^{3}$ Game theoretical models usually exclude behavioral mechanisms by assumption, thus the points of visibility and recognition cannot play a role there. The same holds for being judged incorrectly by people from outside their own field. Signaling through first authorship can, however, play a role. Engers et al. (1999) and Ackerman and Brânzei (2016) provide such theoretical models (see also Joseph et al., 2005). Even the conclusions of these models are ambiguous with respect to the question of which way of ordering authors should be the norm.
} 
focus is naturally reflected in this survey, but the results are similarly relevant for other fields. ${ }^{4}$

If alphabetical name ordering gives an advantage to some authors over others, it is clearly unfair. In addition, it can also be inefficient. Such discrimination may for example hinder the best people from occupying the best positions. This norm is also inefficient if it leads to researchers not choosing the collaborators they otherwise would. $^{5}$

Previewing the results, there is convincing evidence that alphabetical discrimination exists. Many of the reported findings are statistically significant. However, it is worth noting that also the direction of the non-significant findings almost always points toward alphabetical discrimination. As there is a wide variety of different outcomes examined, it is not surprising that the estimated quantities are very different. They reach from an estimated $5 \%$ increase in probability to receive tenure at a top-35 economics department in the US for researchers with an A-name as compared to researchers with a Z-name up to an estimated $60-70 \%$ more downloads for researchers on RePEC (in the top-1000). There is also evidence that researchers perceive alphabetical discrimination to exist and react to it strategically. This happens in various ways, from researchers with names late in the alphabet shying away from collaborations to researchers manipulating their last names.

\footnotetext{
${ }^{4}$ It is also worth noting that it is possible to have a mix of contribution-based and alphabetical ordering. Mongeon et al. (2017) find that in the biomedical literature researchers sometimes use a contributionbased order for primary and supervisory authors (first authors and last authors) and an alphabetical order for middle authors (in particular when the number of co-authors is very large). Such an ordering is in spirit much closer to a contribution-based order than to an alphabetical order, because the alphabetical order appears only in the least important middle positions. The rest of this survey will not address such variants of name ordering as these are not of great importance in almost all studies that this survey is based on (which are from economics and related social sciences).

${ }^{5}$ One can argue that the absence of conflicts over first authorship is efficient under an alphabetical norm. However, given that contribution-based name ordering is the standard in the vast majority of fields and given that there is more and more interdisciplinary collaboration (e.g., Morillo et al., 2003) using an alphabetical norm in some fields will lead to conflicts over author ordering in interdisciplinary research.
} 
The next section reviews the evidence on the existence of alphabetical discrimination. Section 3 reviews the evidence on researchers' strategical reactions to an alphabetical norm. Section 4 concludes.

\section{Are Authors with Last Names Early in the Alphabet Given an Advantage?}

There is a variety of studies analyzing whether authors with names early in the alphabet have an advantage over other authors when the author ordering norm is alphabetical. Almost all of them investigate whether this is the case in economics. The outcome variables studied reach from receiving tenure at good universities over receiving a Nobel (memorial) prize to numbers of downloads or citations. Some of the studies concentrate on the top performers in a discipline as otherwise a pass-through effect could mask the effects of alphabetical discrimination. ${ }^{6}$ However, concentrating on top performers is not the only possibility to identify alphabetical discrimination; most studies carefully control in one way or another for other factors that could potentially drive the results, as for example the correlation between surname initials and ethnicity.

Whether the likelihood to work at top departments is influenced by surname initials is investigated by Einav and Yariv (2006) and Efthyvoulou (2008). The former examine whether authors with surnames early in the alphabet are more likely to receive tenure at top-economics departments in the US (looking at top-5, top-10, top-20, and top-35 departments). Einav and Yariv (2006) argue that if visibility and recognition play a role, these effects should be (almost) absent at the beginning of careers but become more important thereafter. Therefore, alphabetical discrimination should lead to tenured faculty having on average surnames earlier in the alphabet than untenured faculty. Indeed, this is what they find. The empirical distribution functions of surname initials

\footnotetext{
${ }^{6}$ This pass-through is best explained with a simple example. Assume that the best departments hire the best scholars with names early in the alphabet. Then the faculty of the second best departments may still fail to reflect alphabetical discrimination, because the second best departments may hire the best scholars with names late in the alphabet and the second best scholars with names early in the alphabet.
} 
of tenured faculty lie almost completely to the left of the ones of untenured faculty. Results are only statistically significant for top-5 and top-10 departments. Estimates are that the probability of receiving tenure at these departments are roughly 1 percentage point higher per letter earlier in the alphabet accruing to a difference of about 26 percentage points between someone with an A-surname compared to someone with a Z-surname. The effects are robust to controlling for a variety of different variables. For top-20 and top-35 departments their estimates are smaller and not significant (the estimated probability of receiving tenure is about 5 percentage points higher for A-surnames than for Z-surnames). The authors compare their results to the same analysis in psychology, a field that has many similarities to economics but makes use of the contribution-based norm. The described patterns do not exist in psychology. Furthermore, the authors examine the time-trend of their findings. They argue that, as much work in economics in earlier years was single-authored, this bias should have been weaker in the past if the findings are driven by alphabetical discrimination. This is indeed the case.

The analysis by Einav and Yariv (2006) is extended by Efthyvoulou (2008). He compares the likelihood to work at a top department with the probability of working at a lowranked department. Analyzing the top-17 and bottom-51 departments in the US he finds that earlier surnames make it more likely to work at a top department. His estimate for academic faculty at all ranks is that having an A-surname makes it about $8 \%$ more likely to work at a top department than having a Z-surname. Given that the effects of alphabetical discrimination are more likely to have an effect on older faculty, the same analysis is conducted for full professors. There, the estimate is that it is about $20 \%$ more likely that someone is a full professor at a top department when the surname starts with $\mathrm{A}$ rather than $\mathrm{Z}$. The results for faculty at all ranks are not statistically significant while the results for full professors are marginally significant. The results are robust to controlling for nationality and name origin. The same relationships are then analyzed for the UK, albeit with a much smaller sample, using the top- 6 and bottom-18 departments. Again the results point towards alphabetical discrimination. Having an A-surname rather than a Z-surname makes it about $12 \%$ 
more likely to work at a top department for academics at all ranks and around $35-40 \%$ more likely to work at a top department as a professor or reader.

Other measures of success that depend to a large extent on the perception by others are becoming fellows of the Econometric Society, receiving a Clark Medal or receiving the Nobel Prize. Einav and Yariv (2006) report that A-surnames of top-10 economics departments are more likely than Z-surnames to become fellows of the Econometric Society by about $20 \%$. This effect is marginally significant. When conducting the same analysis for top-35 departments, estimates become smaller (accruing to around 5\% for an $A-Z$ comparison) and insignificant. The relationship between surname initials and receiving a Clark Medal or Nobel Prize point again in the direction of alphabetical discrimination but the numbers of such winners are very low and the results correspondingly insignificant.

A different measure of success is the number of papers published in peer-reviewed journals. Assuming that it is easier to publish for well-known researchers and for researchers at well-respected institutions, alphabetical discrimination should lead to researchers with names early in the alphabet publishing more papers than others (as, e.g., visibility and recognition will lead these researchers to be better-known and to work at better institutions). This is examined by van Praag and van Praag (2008) who call the number of publications "scientific weight" and the number of publications per year "productivity". They consider data from 11 mainstream economics journals. As alphabetical name ordering takes time to have an effect, they consider both the sample of all authors and the sample of established authors. In the sample of established authors they find a significant effect of the scientific weight being higher for authors with names earlier in the alphabet (they do not find an effect in the sample of all authors). They obtain similar results for productivity. According to their results, "a Z-author would deserve a $16 \%$ premium on his observed weight compared with an A-author". Their regressions are robust to employing median regressions instead of OLS. Furthermore, they find that the growth of academic productivity increases faster 
for researchers with surnames early in the alphabet which can be expected if alphabetical discrimination plays a role via visibility and recognition.

Related but different measures of success are the numbers of downloads and abstract views scholars receive on research networks. Efthyvoulou (2008) investigates data from the top-1,000 authors according to downloads on logec.repec.org and from the top-1,000 authors according to abstract views. He finds that on average surnames of top-100 authors are earlier in the alphabet than surname initials of authors ranked 200-300, etc. up to rank 600 after which surname initials stay constant. The author finds that being an A-author rather than a Z-author increases (the logarithm of) file downloads by $13 \%$ and abstract views by $11 \%$. These findings are statistically significant and robust to removing outliers and to controlling for "output" and "productivity" defined as the total number of publications and the average number of publications per year (the author finds no effect of surname initials on output or productivity). Efthyvoulou (2008) furthermore conducts probit regressions regarding the likelihood to be among the highest ranked authors rather than among the lowest ranked authors. The effects are strongly significant and sizeable: the likelihood of being among the highest ranked authors in terms of downloads is almost $60 \%$ higher for an A-author than for a Z-author. For abstract views the corresponding increase in likelihood is almost $40 \%$. When controlling for output or productivity the effect of the surname initial becomes even stronger adding up to about $60-70 \%$ for downloads and $50 \%$ for abstract views.

Empirical evidence of a different kind is provided by Maciejovsky et al. (2009). They conduct a survey experiment among academics in which participants have to assign contribution credits. Participants are shown different pairs of author groups made up of a list of common British names. The order of the names is sometimes alphabetical and sometimes not. The experimenters underline one author in each group (not necessarily placed at the same position) and ask participants for their opinion on which of these authors has contributed more to the respective paper. They do this separately for economics (with alphabetical name ordering), psychology (with contribution-based 
name ordering), and marketing (a discipline without a very strong ordering norm). Not surprisingly, researchers in psychology and marketing assign higher contribution credit to first authors independently of whether names are in alphabetical order or not. Notably, the same holds for economists. Even when names are listed in alphabetical order, economists assign higher contribution credit to earlier authors. ${ }^{7}$

There are also studies examining the alphabetical ordering of reference lists. Such ordering can favor articles with first authors early in the alphabet if people start scanning through the lists from top to bottom with items presented first receiving more attention (this results in alphabetical discrimination under both ordering norms). This furthermore leads to articles with the same first authors appearing in a row leading to more recognition of first authors early in the alphabet (resulting in alphabetical discrimination in particular under an alphabetical norm). Huang (2015) investigates this with a set of about 850000 articles from the Web of Science data base. The author finds that papers with first authors early in the alphabet are cited more often. This effect does not exist when instead considering the last authors of the articles. The author argues that the effect stems indeed from more attention being paid to articles at the top of a reference list as it is correlated with the length of citing papers' reference lists. The effect is stronger in fields that make use of alphabetical name ordering more often and robust to conducting a more sophisticated diff-in-diffin-diff analysis. Arsenault and Larivière (2015) conduct a simple analysis based solely on correlations and find that in most disciplines the uncitedness rate is greater when the last name of the first author comes later in the alphabet. They find a particularly strong effect for the field of mathematics, suggesting that this effect is indeed exacerbated by an alphabetical norm.

It is also sometimes claimed that authors with names late in the alphabet are at a disadvantage in disciplines with a contribution-based norm (e.g. because of the

\footnotetext{
${ }^{7}$ This occurs with two and three authors, while for articles with four authors economists give most contribution credit to the first author followed by the second author and then the last author. This could stem from contribution-based ordering where the last position can play a special role (the "senior" author).
} 
alphabetization of reference lists or because some intentional alphabetical ordering always exists). This is not the focus of this survey. Nevertheless, a study that should be placed in this context by Abramo and D'Angelo (2017) provides some interesting results for this survey. ${ }^{8}$ The authors provide correlations between surname initials and citations per researcher. Mere correlations are not a good tool to identify alphabetical discrimination, but if even this crude measure points toward discrimination it could be seen as a particularly strong sing. The authors consider data on Italian authors from the Web of Science in a variety of fields. Unfortunately, economics is not included in the study. Except for mathematics, all considered fields primarily follow a contributionbased norm (with some intentional alphabetization occurring) and mathematics is grouped together with computer science making up a heterogeneous field in terms of author ordering norms. The correlations between citations and last name initials do not reveal discrimination in the fields with mainly a contribution-based norm. The correlation in the category mathematics and computer science is basically zero (0.004) when looking at all scholars and increases more than tenfold (to 0.057 ) when looking at the top $10 \%$ of professors. As the correlations should be regarded as a lower bound on discrimination and as the effect increases when looking at the better $10 \%$ (which is better suited to identify an effect but still very broad), it seems that even this study (albeit all efforts to find no effect) points toward alphabetical discrimination when interpreted correctly (although these numbers should be interpreted with great care).

\section{Do Researchers React Strategically to an Alphabetical}

\section{Norm?}

Multiple studies investigate whether researchers react to (perceived) alphabetical discrimination. Kadel and Walter (2015) investigate whether the fraction of singleauthored articles is higher for authors with surnames late in the alphabet. They also investigate for co-authored articles whether the fraction of two-authored articles is higher (thus examining whether authors are afraid to disappear in the term "et al.").

\footnotetext{
${ }^{8}$ The methodology used in this article and the drawn conclusions have been heavily criticized (Weber, 2017).
} 
They investigate this for economics and finance, separately. They use data from 11 mainstream economics journals and 10 finance journals and restrict their sample to surnames from $A$ to $E$ and from $U$ to $Z$. They do not find a difference with respect to the fraction of single authored papers, in economics and finance alike. They find a significant difference with respect to the number of two-authored articles in economics. Authors with surnames from A to E write $45 \%$ of co-authored papers in teams of three or more, while only $42 \%$ of authors with surnames from $U$ to $Z$ do so. They do not find such an effect in finance.

Laband (2002) compares authorship in agricultural economics and the rest of economics exploiting that author ordering is usually contribution-based in agricultural economics while it is usually alphabetical in the rest of economics. He hypothesizes that if authors act strategically there should be more collaboration in agricultural economics (in line with the argument that authors late in the alphabet avoid collaboration in the rest of economics). He furthermore hypothesizes that strategic behavior should lead to author groups being larger in agricultural economics (in line with authors late in the alphabet avoiding large author groups in economics not to be pushed back in the author list). ${ }^{9}$ The author considers data from three top journals in economics and three top-field journals in agricultural economics. He finds that more articles are co-authored in agricultural economics than in general economics $(77 \%$ compared to 65\%). This difference is statistically significant. Also the average number of authors per co-authored article is higher in agricultural economics (2.6 compared to 2.3). This difference is also statistically significant.

In a similar vein, Einav and Yariv (2006) consider publications from the top-5 economics journals. They find that authors with surnames late in the alphabet are less likely to cooperate in teams of four or five authors. More precisely, average surname

\footnotetext{
${ }^{9}$ The exact argumentation by Laband (2002) is not the one stated here. The author's argumentation starts out from an "importance on priority". This notwithstanding, the empirical tests of the hypotheses are relevant for this survey.
} 
initials (coded from $A=1$ to $Z=26$ ) are lower for articles with one, two, or three authors than for articles with four or five authors.

Ong et al. (2015) analyze citation data from the top-23 economics journals and from the top-30 management journals from Web of Science (in these data, about $83 \%$ of articles in economics exhibit alphabetical author ordering compared to about $48 \%$ in management). They find that citation ranks in economics are influenced by last name initials. This is not the case in management. The authors find that for single-authored papers articles written by researchers with surnames late in the alphabet are cited more often than articles written by researchers with surnames early in the alphabet. The difference in citation rank amounts to a bit above 30 places out of 1,000 for an article written by a Z-author as compared to an article written by an A-author. When they look at papers with two authors they find exactly the opposite effect of about the same magnitude. The results are robust to various controls. The authors do not find effects for papers with three or four authors. Overall, their results are in line with authors late in the alphabet writing better single-authored papers than authors early in the alphabet due to selection into single-authorship when working on the best ideas. Similar results are not found in management.

Another way to see whether authors react to an alphabetical norm is to investigate whether the likelihood to deviate from this norm is higher for authors late in the alphabet. Einav and Yariv (2006) analyze publications in the top-5 economics journals with respect to this question. Again coding last name initials from $A=1$ to $Z=26$, they find that articles deviating from alphabetical name ordering have higher average surname initials. This is the case for two-, three-, and four-author articles (the findings are only significant for three and four authors). If authors with surnames later in the alphabet are on average not more likely to be greater contributors, the only explanation that rests is that "authors perceive alphabetical discrimination to exist" (Einav and Yariv, 2006) and that they react strategically in response.

A similar but more technical analysis is conducted by van Praag and van Praag (2008). With their data from 11 mainstream economics journals, they estimate the "hurdle" to 
deviate from the alphabetical norm. Their results are that authors later in the alphabet employ on average a lower hurdle to deviate from alphabetical name ordering than authors early in the alphabet. These results are statistically significant.

A different way to identify whether researchers react strategically to alphabetical discrimination is to look at manipulations of last names. Efthyvoulou (2008) considers such manipulations. First, he considers names with prefixes $D$ and $V$ (which are particularly frequent, e.g., "de", "de la", "den", or "van", "van den", "von") and appear in very different positions of the alphabet. Researchers have some leeway in trying to include or exclude such prefixes, for example by writing them in capital or small letters, by including them with the first name, or by putting them behind the last name. The data used are multi-authored on EconLit. Probit regressions suggest that Dauthors are $52 \%$ more likely to use their prefix as part of their last name than Vauthors (when the number of co-authors is controlled for; otherwise the estimate is 33\%). The differences are statistically significant. Other scholars who have some freedom in determining their last names listed on articles are scholars with two last names. Investigating publications from 11 economics journals, Efthyvoulou (2008) finds that $53 \%$ of authors with two last names place the name coming earlier in the alphabet first. The likelihood to have the last name earlier in the alphabet as the first last name increases with the distance between the two names in the alphabet. Having the maximum alphabetical distance between both names (A vs. Z) increases the likelihood of having the earlier last name as first last name by more than $65 \%$ compared to a distance of only one letter (e.g., K vs. L). These differences are statistically significant. Looking at psychology as a comparison without an alphabetical norm, the author does not find these effects. Yet another last name manipulation examined concerns the transcription of Greek names into English. Some Greek letters have more than one possible transcription to English. The Greek letter $\Gamma$ for example can be transcribed as $\mathrm{G}$ or as $\mathrm{Y} .68 \%$ of authors in economics choose the transcription to English with the letter coming earlier in the alphabet compared to $66 \%$ in psychology. This hints at economists being aware of alphabetical discrimination, but the differences are very small. Other factors seem to play a more important role in 
such a decision (some letters may, for example, just look more natural to Greek people as transcriptions).

\section{Discussion}

The evidence provided in a variety of studies leaves no doubt that an alphabetical author ordering norm disadvantages researchers with last names toward the end of the alphabet. There is furthermore convincing evidence that researchers are aware of this and that they react strategically to such alphabetical discrimination, for example with their choices of who to collaborate with.

Given that only a few fields predominantly order authors alphabetically, it may be the easiest solution for these fields to abandon this special arrangement. Instead, the norm prevalent in the vast majority of disciplines may be followed according to which the relative contribution determines the author order. This point is strengthened by the fact that inter-disciplinary collaborations are increasing in importance (e.g., Morillo et al., 2003) where conflicts can arise as a result of different ordering norms in different fields. The point is strengthened further by the fact that in the fields with alphabetical norms these norms are not even necessarily unique (for instance in physics, where only one subfield follows this norm).

An argument that might be brought up in favor of an alphabetical norm is that it supposedly makes it possible to contribute equally to an article while being assigned equal credit. As we have seen, this argument is wrong; credit is actually not equally assigned under such a norm. Moreover, the problem of unequal credit assignment in case of equal contributions can be alleviated under a contribution-based rule. Author groups working together on multiple projects can, for example, alternate the author order. In addition to that, in cases where it is appropriate, I recommend inserting a footnote after some (or all) author names stating "These authors contributed equally to this work. The order of author names was randomly determined."

The suggestion by Levitt and Thelwall (2013) that journals issue "a clear statement ... that this practice [intentional alphabetical name ordering] is unacceptable" would 
certainly help to abolish the norm. Of course, there are also other possibilities to help reducing alphabetical discrimination. References could be ordered according to their order of appearance in the text as is already done in some disciplines. This seems to be a good idea independent of what the author ordering norm is. Not referring to articles in the text as "first author et al." but instead with all names written out the first time and last name initials in the remainder (as also partly used already) would probably also reduce alphabetical discrimination. Nevertheless, as the vast majority of scientific disciplines rely on a contribution-based norm, the most reasonable solution seems to be for the few disciplines with an alphabetical norm to get rid of this special arrangement.

While it has been suggested many times to abolish alphabetical name ordering, not much is known about how this can best be achieved. Some of the suggestions call upon researchers to change their behavior and disregard the norm, other suggestions call upon professional associations and journals to discourage the use of such a practice. Which of the suggestions would work well to put an end to alphabetical ordering is not clear. This is especially the case as the political economy of changing such a norm is not trivial. Not only young researchers with names early in the alphabet benefit from sticking with an alphabetical norm but also old researchers with names late in the alphabet who may be afraid that their contribution to the articles written under the alphabetical norm may be discounted after a norm change. On the other hand, not only young researchers with names late in the alphabet would profit from a change but also researchers with names early in the alphabet at later stages of their career.

\section{References}

Abramo, G., \& D'Angelo, C. A. (2017). Does your surname affect the citability of your publications? Journal of Informetrics, 11(1), 121-127.

Abt, H. (1992). Publication practices in various sciences. Scientometrics, 24(3), 441447. 
Ackerman, M., \& Brânzei, S. (2016). The authorship dilemma: Alphabetical or contribution? Autonomous Agents and Multi-Agent Systems, 1-17.

Arsenault, C., \& Larivière, V. (2015). Is paper uncitedness a function of the alphabet? Proceedings of the 15th International Conference of the International Society for Scientometrics and Informetrics, 286-287.

Banerjee, A., Mookherjee, D., Munshi, K., \& Ray, D. (2001). Inequality, control rights, and rent seeking: Sugar cooperatives in Maharashtra. Journal of Political Economy, 109(1), 138-190.

Engers, M., Gans, J. S., Grant, S., \& King, S. P. (1999). First-author conditions. Journal of Political Economy, 107(4), 859-883.

Efthyvoulou, G. (2008). Alphabet economics: The link between names and reputation. The Journal of Socio-Economics, 37(3), 1266-1285.

Einav, L., \& Yariv, L. (2006). What's in a surname? The effects of surname initials on academic success. The Journal of Economic Perspectives, 175-188.

Frandsen, T. F., \& Nicolaisen, J. (2010). What is in a name? Credit assignment practices in different disciplines. Journal of Informetrics, 4(4), 608-617.

Garfield, E. (1997). All sorts of authorship. Nature, 389(6653), 777-777.

Gazni, A., \& Didegah, F. (2011). Investigating different types of research collaboration and citation impact: a case study of Harvard University's publications. Scientometrics, $87(2), 251-265$.

Goldstein, D. G., \& Gigerenzer, G. (2002). Models of ecological rationality: the recognition heuristic. Psychological review, 109(1), 75.

Huang, W. (2015). Do ABCs Get More Citations than XYZs?. Economic Inquiry, 53(1), 773-789. 
Hudson, J. (1996). Trends in multi-authored papers in economics. The Journal of Economic Perspectives, 153-158.

Joseph, K., Laband, D. N., \& Patil, V. (2005). Author order and research quality. Southern Economic Journal, 545-555.

Kadel, A., \& Walter, A. (2015). Do scholars in Economics and Finance react to alphabetical discrimination? Finance Research Letters.

Laband, D. N. (2002). Contribution, attribution and the allocation of intellectual property rights: economics versus agricultural economics. Labour Economics, 9(1), 125-131.

Lake, D. A. (2010). Who's on first? Listing authors by relative contribution trumps the alphabet. PS: Political Science \& Politics, 43(01), 43-47.

Levitt, J. M., \& Thelwall, M. (2013). Alphabetization and the skewing of first authorship towards last names early in the alphabet. Journal of Informetrics, 7(3), 575-582.

Maciejovsky, B., Budescu, D. V., \& Ariely, D. (2009). The researcher as a consumer of scientific publications: How do name-ordering conventions affect inferences about contribution credits? Marketing Science, 28(3), 589-598.

Mongeon, P., Smith, E., Joyal, B., \& Lariviere, V. (2017). The rise of the middle author: Investigating collaboration and division of labor in biomedical research using partial alphabetical authorship. Working Paper.

Morillo, F., Bordons, M., \& Gómez, I. (2003). Interdisciplinarity in science: A tentative typology of disciplines and research areas. Journal of the American Society for Information Science and technology, 54(13), 1237-1249.

Ong, D., Chan, H. F., Torgler, B., \& Yang, Y. A. (2015). Endogenous selection into single and coauthorships by surname initials in economics and management. Working Paper.

Over, R., \& Smallman, S. (1973). Maintenance of individual visibility in publication of collaborative research by psychologists. American Psychologist, 28(2), 161. 
Ray, D., \& Robson, A. (2016). Random order: Or, how to co-author if you must. Working Paper.

Rudd, E. (1977). The effect of alphabetical order of author listing on the careers of scientists. Social Studies of Science, 7(2), 268-269.

Sarsons, H. (2015). Gender differences in recognition for group work. Working Paper.

Serwe, S., \& Frings, C. (2006). Who will win Wimbledon? The recognition heuristic in predicting sports events. Journal of Behavioral Decision Making, 19(4), 321-332.

Shevlin, M., \& Davies, M. N. (1997). Alphabetical listing and citation rates. Nature, 388(6637), 14-14.

Stubbs, C. (1997). The serious business of listing authors. Nature, 388(6640), 320-320.

Tregenza, T. (1997). Darwin a better name than Wallace? Nature, 385(6616), 480-480.

Van Praag, C. M., \& van Praag, B. (2008). The benefits of being economics professor A (rather than Z). Economica, 75(300), 782-796.

Waltman, L. (2012). An empirical analysis of the use of alphabetical authorship in scientific publishing. Journal of Informetrics, 6(4), 700-711.

Weber, M. (2017). Comment on "Does your surname affect the citability of your publications". Journal of Informetrics. 11(3):835-837.

Wuchty, S., Jones, B. F., \& Uzzi, B. (2007). The increasing dominance of teams in production of knowledge. Science, 316(5827), 1036-1039.

Zuckerman, H. A. (1968). Patterns of name ordering among authors of scientific papers: A study of social symbolism and its ambiguity. American Journal of Sociology, 276-291. 